This document is the accepted manuscript version of the following article:

\title{
Durchblick mit Holografie
}

\section{Erwin Hack | Lorenzo Valzania | Peter Zolliker}

\section{Vorspanntext:}

Bildgebung mit Terahertzstrahlung basiert bislang auf Abscannen eines Objekts mit einem Detektor. Mikrobolometer-Kameras ermöglichen nun eine Bildgebung wie mit jeder Kameratechnik. Flächendetektoren erlauben überdies den Einsatz holografischer Methoden. Trotz der großen Wellenlänge reicht die dreidimensionale Ortsauflösung für Anwendungen in der Biomechanik.

Es ist ein alter Menschheitstraum, durch Wände oder andere Materialien hindurchschauen zu können. Dieser Traum wurde vor über hundert Jahren erstmals verwirklicht, als Wilhelm Conrad Röntgen hochenergetische Strahlen dazu verwendete, die innere Struktur eines Körpers bildlich darzustellen. Das Durchleuchten mit Röntgenstrahlen hat sich in Medizin und Technik inzwischen zu hochauflösenden bildgebenden Verfahren weiterentwickelt. Allerdings ist ein gewichtiger Nachteil die Strahlenbelastung und deren mögliche schädigenden Einflüsse auf das untersuchte biologische oder technische Objekt.

Andere Methoden zum Erfassen von inneren Strukturen, insbesondere in Medizinanwendungen, beruhen auf Magnetresonanz oder Ultraschall. Während für die Magnetresonanz in der Regel ein hoher apparativer Aufwand betrieben werden muss, ist die Ultraschalltechnik relativ einfach handzuhaben. Interessanterweise gewähren viele Objekte auch einen Einblick in ihr Innenleben, wenn man im elektromagnetischen Spektrum von den Röntgenstrahlen zu Terahertz ( $\mathrm{THz}$ )-Strahlen geht, also von Wellenlängen unterhalb zehn Nanometer zu Wellenlängen zwischen 0,03 bis 0,3 mm. Weil in diesem Wellenlängenbereich viele nichtleitende Materialien keine Strahlung mehr absorbieren, werden sie "durchsichtig". So kann THz-Strahlung Holz, viele Kunststoffe, Keramiken und Halbleiter, aber auch Textilien und Papier durchdringen [1]. Und sie gilt als harmlos für den menschlichen Organismus.

Diese Eigenschaft hat dazu geführt, dass THz-Strahlung im Sicherheitsbereich eingesetzt wird, sei es um unter der Kleidung versteckte Gegenstände aufzuspüren ("Nacktscanner") oder den Inhalt von Gepäckstücken zu untersuchen. Auch medizinische Anwendungen sind so möglich, wie die berührungsfreie Untersuchung von Hautkrebs, Gefäßen oder der Wundheilung durch einen Verband hindurch [2]. Allerdings sind diese Anwendungen auf oberflächennahe 
Bereiche beschränkt, da Wasser die THz-Strahlung stark absorbiert und reflektiert. Im technischen Bereich und der zerstörungsfreien Prüfung steht die Untersuchung von beschichteten Objekten im Vordergrund, zum Beispiel lackierte Tabletten, Autotüren oder hinter Gips verborgene Gemälde [3]. Auch die Qualitätskontrolle von Produkten, die in Plastik oder Karton verpackt sind, ist möglich.

\section{THz-Quellen und THz-Detektoren}

THz-Strahlen werden von jedem Gegenstand abgestrahlt, da sie ein Teil der natürlichen Wärmestrahlung sind. Diese passive Strahlung kann gemessen werden. Weil sie aber sehr schwach ist, bietet sie nur dann Vorteile gegenüber der mit Wärmebildkameras aufgenommenen Infrarot (IR)-Strahlung, wenn letztere auf dem Weg zur Kamera absorbiert wird. Richtig interessant werden Anwendungen dann, wenn THz-Strahlungsquellen den Gegenstand aktiv beleuchten oder durchstrahlen können.

Heutzutage stehen verschiedene THz-Quellen für Industrie- und Labor-Anwendungen zur Verfügung, die ganz unterschiedliche physikalischen Erzeugungsmechanismen nutzen (Tabelle 1, [1]). Durchstimmbare und gepulste THz-Quellen werden in spektroskopischen Verfahren eingesetzt, um chemische Informationen über ein Material zu erhalten oder um aus der Laufzeit Schichtdicken zu bestimmen. Die erzeugte Strahlung dieser THz-Quellen ist heute noch so schwach, dass meist nur einzelne, großflächige Detektorelemente zum Nachweis verwendet werden können. Entsprechend muss eine bildgebende Darstellung des Objekts durch Rastern erfolgen [4]. Damit wird die Aufnahmezeit für ein Bild lang, und eine Messung dynamischer Vorgänge ist schwierig.

Mit Quantenkaskaden- und Gaslasern steht hingegen eine ausreichende THz-Leistung für die Verwendung von Flächendetektoren zur Verfügung. Zum Einsatz kommen dabei pyroelektrische Detektoren, deren Pixelzahl heute auf circa 320 × 320 beschränkt ist. Der pyroelektrische Effekt beschreibt die Eigenschaft einiger piezoelektrischer Kristalle, auf eine zeitliche Temperaturänderung mit einer Ladungstrennung zu reagieren. Er erfordert bei solchen Kameras eine periodische Modulation des Signals von einigen Hertz, was die Messung dynamischer Vorgänge behindert.

In den letzten Jahren sind Mikrobolometer-Kameras interessant geworden, weil sie als kostengünstige Infrarotkameras immer verbreiteter sind, und dennoch eine ausreichende Empfindlichkeit auf THz-Strahlung aufweisen [5]. Dazu muss ein THz-transparentes Schutzglas vor dem Sensor sitzen. Ihre Wirkungsweise beruht auf der Widerstandsänderung einer Membran durch die Wärme- oder in unserem Fall die THz-Strahlung. Matrixgrößen von 480 x 640 Pixeln mit 17 bis $25 \mu \mathrm{m}$ Pixelabstand sind heute Standard. Weil die Mikrobolometer-Kamera auf Wärmestrahlung und THz-Strahlung anspricht, muss erstere als Hintergrundbild abgezogen werden. Dies kann mit einer schaltbaren Blende realisiert werden.

\section{Bildgebung mit Flächendetektoren}

Durch die Verfügbarkeit von ausreichend starken Quellen und empfindlichen Kameras kann THz-Imaging wie eine "gewöhnliche" Bildtechnik behandelt werden. Angepasst an die THz- 
Wellenlängen finden Linsen aus speziellen Materialien wie Silizium oder THz-transparenten Kunststoffen Verwendung, die Abbildungsgeometrie entspricht jedoch derjenigen von Digitalkameras für sichtbares Licht und Wärmebildkameras für IR-Licht. Weil aber die THzWellenlänge die von sichtbarem Licht um das Hundertfache übertrifft, ist bei gleicher Abbildungsgeometrie auch die physikalische Auflösungsgrenze hundertmal geringer.

Um diese Ortsauflösung zu verbessern, stehen verschiedene - von der Optik und Röntgentechnik inspirierte - Verfahren zur Verfügung, die ohne Abbildungslinse auskommen. Bei der digitalen Holografie (Abbildung 1 links), wird das ganze Objekt mit einem kohärenten THz-Strahl beleuchtet. Die am Objekt in Transmission oder Reflexion gestreute Strahlung wird nicht mit einer Linse gesammelt und abgebildet, sondern direkt vom Detektor aufgezeichnet. Ein solches Bild ist also nichts anderes als die Intensitätsverteilung der am Objekt gestreuten THz-Welle. Die Ausbreitung der THz-Welle vom Objekt zum Detektor folgt dem Huygens-Prinzip, jeder beleuchtete Objektoberflächenpunkt trägt somit eine Kugelwelle zur Intensität in jedem Pixel des digital aufgenommenen Bilds bei. Überdies ist auch die Phasenbeziehung zwischen den Pixeln eindeutig festgelegt, weil die THz-Strahlung kohärent ist, Somit enthält das Bild auch eine Phaseninformation, wie sie für ein Hologramm benötigt wird.

Umgekehrt können wir in der Detektorebene jedes Pixel des digitalen Bilds als Ursprung einer Kugelwelle betrachten, deren rechnerische Überlagerung im richtigen Abstand das Objekt rekonstruiert. Die Betrachtungsrichtung kann deshalb umgedreht werden. Das heißt, wir können die aufgezeichnete THz-Welle rechnerisch vom Detektor zurück in Richtung Objekt laufen lassen [6]. Dazu muss aber in jedem Bildpunkt neben der Intensität der gestreuten Welle auch ihre Phase bekannt sein.

Für ein Hologramm benötigen wir also noch die Phaseninformation. Diese können wir mit Hilfe eines überlagerten Referenzstrahls bestimmen, der unter einem durch die Aufnahmegeometrie bedingten Winkel schräg auf den Detektor trifft (Abbildung 1 links). Weil die THz-Wellenlänge die Pixelgröße des Detektors übersteigt, können wir viel größere Einstrahlwinkel als mit sichtbarem Licht verwenden, was den Aufbau vereinfacht. In der Detektorebene interferiert der Referenzstrahl mit der Objektwelle, sodass wir in jedem Punkt die relative Phase zwischen Objekt- und Referenzstrahl bestimmen können. Betrachten wir letzteren als ebene Welle, ist die Phasendifferenz identisch mit der Objektphase - bis auf eine übers Bild linear zunehmende Abweichung, die durch den Einfallswinkel des Referenzstrahls bedingt ist.

Die Holografie stößt allerdings an ihre Grenzen, wenn der Referenzstrahl nicht am Objekt vorbei auf den Detektor gerichtet werden kann, weil das Objekt zu groß oder zu nahe am Detektor ist. In solch einem Fall wird eine andere Technik interessant: die Ptychografie (Abbildung 1 rechts). Auch sie verwendet die vom Objekt gestreute Strahlung, verzichtet aber auf den Referenzstrahl zur Phasenbestimmung. Um für eine holografische Abbildung eine Phaseninformation zu gewinnen, muss man zu anderen Tricks greifen: Die Beleuchtung erhält eine Struktur, das Objekt wird in seiner Ebene verschoben, und es wird an mehreren 
überlappenden Positionen je eine Messung gemacht. In der Gesamtschau kann man aus diesen Aufnahmen die Phase rechnerisch iterativ bestimmen [7]. Dieses Verfahren ist numerisch zwar etwas aufwendiger als die digitale Holografie, dafür sind aber Aufnahmen an einem bewegten Objekt möglich.

Wie schon angedeutet, kann man aus der Amplituden- und Phaseninformation in der Detektorebene das Objekt durch Rückrechnen darstellen. Abbildungen 2a und $2 \mathrm{~d}$ zeigen ein Hologramm und die entsprechende Phase in der Detektorebene nach Reflexion von einem metallischen Objekt. Das Rückrechnen von der Detektorebene in den Objektraum lässt das Objekt erscheinen (Abbildungen 2b, e), allerdings verdreht, weil Objekt- und Detektorebene nicht parallel stehen. Erst die Projektion auf eine entsprechend gedrehte Objektebene ergibt eine befriedigende numerische Rekonstruktion (Abbildungen $2 c, f$ ).

Die Ortsauflösung wird durch verschiedene Faktoren bestimmt (siehe „Auflösungsfaktoren" auf S. Die Numerische Apertur, die bei einer Abbildung mit einer Linse im Wesentlichen durch das Verhältnis von Linsendurchmesser zum Objektabstand bestimmt ist, hat ihre Entsprechung in der Holografie im Verhältnis von Detektordurchmesser zum Objektabstand. Statt teure Mikroskopobjektive einzusetzen, genügt es in der Holografie also, das Objekt näher an den Detektor zu rücken, um die Ortsauflösung zu erhöhen. Darüber hinaus kann man die Detektorfläche künstlich vergrößern, indem man den Detektor in seiner Ebene verschiebt und die Hologramme entsprechend zusammensetzt [8] (Abbildung 1 links). Dieses Verfahren wird Synthetische Apertur genannt [9].

Neben der seitlichen Ortsauflösung ist auch die Auflösung in Strahlrichtung wichtig. Bei Messung in Transmission wird damit das Objekt in seiner Dicke erfasst, bei Messung in Reflexion sein Oberflächenprofil. Der natürliche Maßstab bleibt zwar die Wellenlänge der verwendeten Strahlung. Mit den oben beschriebenen Verfahren der Holografie und Ptychografie kann die Objektphase viel genauer als auf $2 \pi$ (eine Wellenlänge) bestimmt werden. Bei THz-Anwendungen wurden so Auflösungen in Strahlrichtung von rund einem Dreißigstel der Wellenlänge erreicht.

Abbildung 3 zeigt die Verwendung von THz-Holografie in Reflexion, um das Profil einer

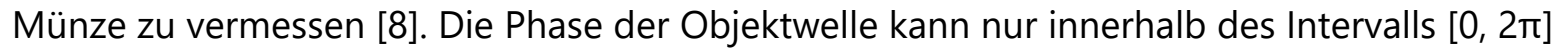
rad, also modulo $2 \pi$, bestimmt werden. Deshalb weist sie jedes Mal einen Sprung von $2 \pi$ rad auf 0 rad auf, wenn der Wegunterschied ein Vielfaches der Wellenlänge beträgt. Um das kontinuierliche Oberflächenprofil zu erhalten, muss man diese Phasensprünge im Bild detektieren und in jedem Punkt ein entsprechendes Vielfaches von $2 \pi$ rad zum gemessenen Phasenbild addieren.

Schließlich ist noch die zeitliche Auflösung zu beachten. Obwohl die MikrobolometerKameras Bildfrequenzen von $50 \mathrm{~Hz}$ erreichen können, ist eine so schnelle THz-Aufnahme in der Regel nicht möglich. Weil die Kamera vor allem auf Wärmestrahlung im Bereich 8-12 $\mu \mathrm{m}$ empfindlich ist, muss regelmäßig ein IR-Hintergrundbild erfasst und subtrahiert werden. Bei 
schwachen Signalen muss das THz-Bild zusätzlich gemittelt werden. Bildfrequenzen von 20 $\mathrm{Hz}$ oder mehr wurden aber schon demonstriert [10,11].

\section{Anwendungen}

Die meisten bildgebenden Anwendungen von THz-Strahlung basieren heute noch auf Rasterverfahren, wobei wahlweise das Objekt oder der Strahl bewegt wird. In unserem Labor hingegen verwenden wir eine Mikrobolometer-Kamera zusammen mit einem THz-Gaslaser. Damit untersuchen wir die biomechanische Kontaktfläche zwischen der Haut und verschiedenen Oberflächen - und deren Reibung. Uns interessiert besonders, wie groß die effektive Kontaktfläche genau ist, weil die Haut wegen ihrer Struktur nicht vollflächig aufliegt. Dabei beschäftigt uns auch die Frage, ob und wo in der Kontaktfläche Wasser vorkommt. Denn sowohl die Druck- als auch die Feuchteverteilung bestimmen die Reibung mit. Solche Fragestellungen sind interessant für viele Gebiete, vom Wundliegen bis zur Sportbekleidung.

Für solche Untersuchungen müssen wir durch ein THz-transparentes Deckmaterial hindurch messen, um an die Kontaktfläche heranzukommen. Das erste Problem, das es dabei zu lösen gilt, ist die vom Deckmaterial verursachte Streuung der THz-Strahlung. Auch wenn dieses transparent für THz-Strahlen ist, wird an der Grenzfläche wegen des höheren Brechungsindexes ein Teil dieser Strahlung reflektiert.

Zur Illustration zeigt Abbildung 4a einen metallischen Siemens-Stern hinter einer optisch undurchsichtigen, aber THz-transparenten Platte aus Teflon. Im Hologramm (Abbildung 4b) treten überlagert zur gestreuten Objektwelle Interferenzstreifen auf, die von der Teflonplatte herrühren. Wenn dann noch der Referenzstrahl überlagert wird, besteht das Hologramm aus der Interferenz von drei Beiträgen: vom interessierenden Objekt, von der Platte und vom Referenzstrahl (Abbildung 4c). Durch Separation der einzelnen Beiträge und nach Kompensation des Plattenbeitrags lässt sich das Objekt rekonstruieren (Abbildung 4d) [12].

Überdies haben wir nachgewiesen, dass sowohl die mit der THz-Holografie erreichbare Ortsauflösung als auch die Höhenauflösung ausreichen, um einen Fingerabdruck zu rekonstruieren. Abbildungen $5 a$ und $5 b$ zeigen einen Fingerkuppen-Abguss und das rekonstruierte THz-Phasenbild. Im tieffrequenten Anteil des Phasenbilds ist die Wölbung der Kuppe als "Höhenlinien" sichtbar (Abbildung 5c), während im hochfrequenten Anteil die durch die Rillen des Fingers verursachte Feinstruktur deutlich erkennbar ist (Abbildung $5 d$ ).

Wir können mit dieser Methode also nicht nur das Oberflächenprofil von metallischen Gegenständen wie der Münze der Abbildung 3 bestimmen, sondern auch von diffus reflektierenden Oberflächen wie der Haut. In unserer Forschung wollen wir nun im nächsten Schritt die effektive Kontaktfläche zwischen Finger und Teflonplatte und dann zwischen Haut und Textilien vermessen.

Dieses Beispiel unterstreicht die Verwendbarkeit der Methode zur Untersuchung von Objekten oder Oberflächen hinter THz-transparentem Material. Neben den medizinischen Anwendungen sind auch Einsätze in der industriellen Inspektion denkbar, etwa zur Analyse 
von Gütern in Plastikverpackungen oder zum Nachweis von elektronischen Bauteilen unter einer Vergussmasse.

\section{Zusammenfassung}

Bildgebung mit Terahertzstrahlung basiert bislang auf Abscannen eines Objekts mit einem Detektor. Mikrobolometer-Kameras ermöglichen nun eine Bildgebung wie bei einer normalen Kameratechnik. Das erlaubt auch den Einsatz holografischer Methoden. Bei ausreichend Platz, um einen Referenzstrahl seitlich in die Kamera einzustrahlen, ist herkömmliche digitale Holografie möglich. Ist es dafür zu eng, lässt sich über die Methode der Ptychografie holografische Information gewinnen. Trotz der großen $\mathrm{THz}$-Wellenlänge reicht die dreidimensionale Ortsauflösung für Anwendungen in der Biomechanik. So lässt sich die Kontaktfläche einer Fingerkuppe mit dem Untergrund genau untersuchen.

\section{Stichwörter}

Terahertz-Strahlung, Mikrobolometer-Kamera, THz-Holografie, THz-Ptychografie, Oberflächenstruktur, Kontaktfläche, Fingerkuppe.

\section{Danksagung}

Wir danken dem Schweizerischen Nationalfonds zur Förderung der wissenschaftlichen Forschung für die unter der Projektnummer 200021_160078 gewährte Finanzierung.

\section{Literatur}

[1] S. S. Dhillon et al., J. Phys. D: Appl. Phys. 2017, 50, 043001.

[2] Q. Sun et al., Quant. Imaging Med. Surg. 2017, 7, 345.

[3] X. C. Zhang, J. Xu, THz-technology in nondestructive evaluation, Introduction to $\mathrm{THz}$ Wave Photonics, Springer US, New York 2010, S. 175.

[4] M. Theurer et al., Phys. Unserer Zeit 2009, 39(6), 296.

[5] E. Hack et al., Sensors 2016, 16, 221.

[6] U. Schnars, J. Opt. Soc. Am. A. 1994, 11, 2011.

[7] L. Valzania et al., Opt. Lett. 2018, 43, 543.

[8] P. Zolliker, E. Hack, Opt. Express 2015, 23, 10957.

[9] H. Huang et al., Appl. Opt. 2016, 55, A43.

[10] A. W. M. Lee et al., IEEE Phot. Tech. Lett. 2006, 18, 1415.

[11] X. Zheng et al., J. Infrared, Millim. Terahertz Waves 2016, 37, 965.

[12] L. Valzania, P. Zolliker, E. Hack, Optics Express 2017, 25, 11038. 


\section{Die Autoren}

Erwin Hack ist Senior Scientist an der Empa und Dozent an der ETH Zürich. Er studierte Theoretische Physik und promovierte in Physikalischer Chemie über zeitaufgelöste Laserspektroskopie. Sein Forschungsinteresse sind bildgebende Verfahren, darunter SpeckleInterferometrie und Terahertz-Imaging.

Lorenzo Valzania hat ein Diplom in Ingenieurphysik und macht derzeit seine Doktorarbeit. Er forscht mit THz-Imaging an biomechanischen Grenzflächen, insbesondere der Wechselwirkung zwischen Textilien und Haut. Sein weiteres Interesse gilt bildgebenden optischen Verfahren, der Röntgentomografie und THz-Spektroskopie.

Peter Zolliker hat Physik studiert und in Kristallografie promoviert. Nach einem Postdoc am Brookhaven National Laboratory, USA, arbeitete er zunächst in der Firma Gretag Imaging. An der Empa forschte er erst auf dem Gebiet der Bildqualität und Psychophysik; aktuell konzentriert er sich auf THz-Imaging, Holografie und Spektroskopie.

\section{Anschrift}

Dr. Erwin Hack, Empa, Abteilung Transport at Nanoscale Interfaces, Überlandstrasse 129, 8600 Dübendorf, Schweiz. erwin.hack@empa.ch, lorenzo.valzania@empa.ch, peter.zolliker@empa.ch www.empa.ch/web/s405/measurement_technology

\section{((Infokasten:)) Auflösungsfaktoren}

Die Ortsauflösung von bildgebenden Verfahren beeinflussen verschiedene Faktoren. Die Wellenlänge des verwendeten Lichts ist sozusagen der Maßstab bei der Abbildung. Die Ortsauflösung lässt sich nur schwer unter die Wellenlänge drücken. Die Numerische Apertur beschreibt den Raumwinkel, aus dem die Strahlung zur Abbildung verwendet werden kann. Bei der Abbildung mit einer Linse ist dieser Winkel bestimmt durch den Blendendurchmesser und den Objektabstand, bei der Holografie durch die Detektorfläche und den Objektabstand. Die Pixelgröße mittelt das Bild bei einer Abbildung mit einer Linse sowie das Hologramm bei der digitalen Holografie.

Bei der digitalen Holografie mit einer Wellenlänge $\lambda$, einem quadratischen Flächendetektor mit $N$ Pixeln der Größe $p$ in der Breite und einem Abstand $g$ vom Objekt zum Detektor beträgt die Ortsauflösung $d$ $d=\lambda \frac{g}{N p}$.

\section{TAB. 1 QUELLEN FÜR THZ-STRAHLUNG UND IHRE EIGENSCHAFTEN}

\begin{tabular}{|l|l|l|c|}
\hline THz-Quelle & Erzeugungsprinzip & Spektrale Charakteristik & Leistung $^{1}$ \\
\hline Gaslaser & Rotationsübergang in einem & einzelne, wählbare & $100 \mathrm{~mW}$ \\
\hline
\end{tabular}




\begin{tabular}{|c|c|c|c|}
\hline & organischen Molekül & $\begin{array}{l}\text { Wellenlängen, } \\
\text { Dauerstrichbetrieb }\end{array}$ & \\
\hline $\begin{array}{l}\text { QCL (Quanten- } \\
\text { kaskadenlaser) }\end{array}$ & $\begin{array}{l}\text { elektronischer Übergang in } \\
\text { einer mehrschichtigen } \\
\text { Mikrostruktur }\end{array}$ & $\begin{array}{l}\text { feste Wellenlänge in } \\
\text { mehreren Linien, } \\
\text { Pulsbetrieb }\end{array}$ & $2 \mathrm{~mW}$ \\
\hline $\begin{array}{l}\text { Backward-Wave } \\
\text { Oszillator }\end{array}$ & $\begin{array}{l}\text { Verstärkung eines } \\
\text { Radiofrequenz-Signals in } \\
\text { einer Vakuumröhre }\end{array}$ & $<1,2 \mathrm{THz}$ & $1 \mathrm{~mW}$ \\
\hline Gunn-Diode & $\begin{array}{l}\text { Elektrische Feldausbreitung in } \\
\text { einem Halbleiterelement mit } \\
\text { negativem Widerstand }\end{array}$ & & \\
\hline $\begin{array}{l}\text { Optische } \\
\text { Antenne }\end{array}$ & $\begin{array}{l}\text { Anregung einer elektrischen } \\
\text { Dipolantenne mit einem } \\
\text { kurzen Laserpuls }\end{array}$ & $\begin{array}{l}\text { Breitbandiges Spektrum, } \\
\text { Pulsbetrieb, } \\
\text { typ. 0,1 bis } 7 \mathrm{THz}\end{array}$ & $0,1 \mathrm{~mW}$ \\
\hline $\begin{array}{l}\text { Optische } \\
\text { Mischung }\end{array}$ & $\begin{array}{l}\text { Verstärkung der } \\
\text { Differenzfrequenz von zwei } \\
\text { Lasern in einem nicht- } \\
\text { linearen optischen Kristall }\end{array}$ & $\begin{array}{l}\text { Durchstimmbar, } \\
\text { Pulsbetrieb } \\
0,3-18 \mathrm{THz}\end{array}$ & $0,01 \mathrm{~mW}$ \\
\hline
\end{tabular}

${ }^{1}$ Typische mittlere Strahlungsleistung kommerzieller Systeme. 


\section{((Abbildungsunterschriften))}

\section{ABB. 1 TERAHERTZ-HOLOGRAFIE}

Links: Digitale Holografie in Transmission, mit der Methode der Detektorverschiebung zur Erweiterung der Aufnahmefläche. Rechts: Ptychografie. Das Testobjekt ist jeweils ein Siemens-Stern.

\section{ABB. 2 THz-HOLOGRAFIE IN REFLEXION}

a) Intensität des Streufeldes des Objekts (Siemens-Stern) bei einem Hologramm, b) rekonstruiertes Objekt parallel zum Detektor, c) rekonstruiertes Objekt in der Objektebene, d)-f) entsprechende Phasenbilder.

\section{ABB. 3 THz-HOLOGRAFIE IN REFLEXION}

a) Fünfrappen-Münze, b) rekonstruierte Objektamplitude, c) rekonstruierte Objektphase und d) aus der Phase berechnetes Oberflächenprofil.

\section{ABB. 4 THz-HOLOGRAFIE IN TRANSMISSION}

Siemens-Stern hinter einer THz-transparenten Teflonplatte: a) Schema, b) Hologramm von Objekt und Platte, c) Überlagerung von Objekt und Referenzstrahl, d) Rekonstruktion des Objekts nach Kompensation der Platte.

\section{ABB. 5 HAUTKONTAKT}

a) Fingerkuppen-Profil, b) rekonstruiertes THz-Phasenbild, c) tief- und d) hochfrequenten Profilanteile. 
Referenzstrahı

\section{Detektor}

- Empa
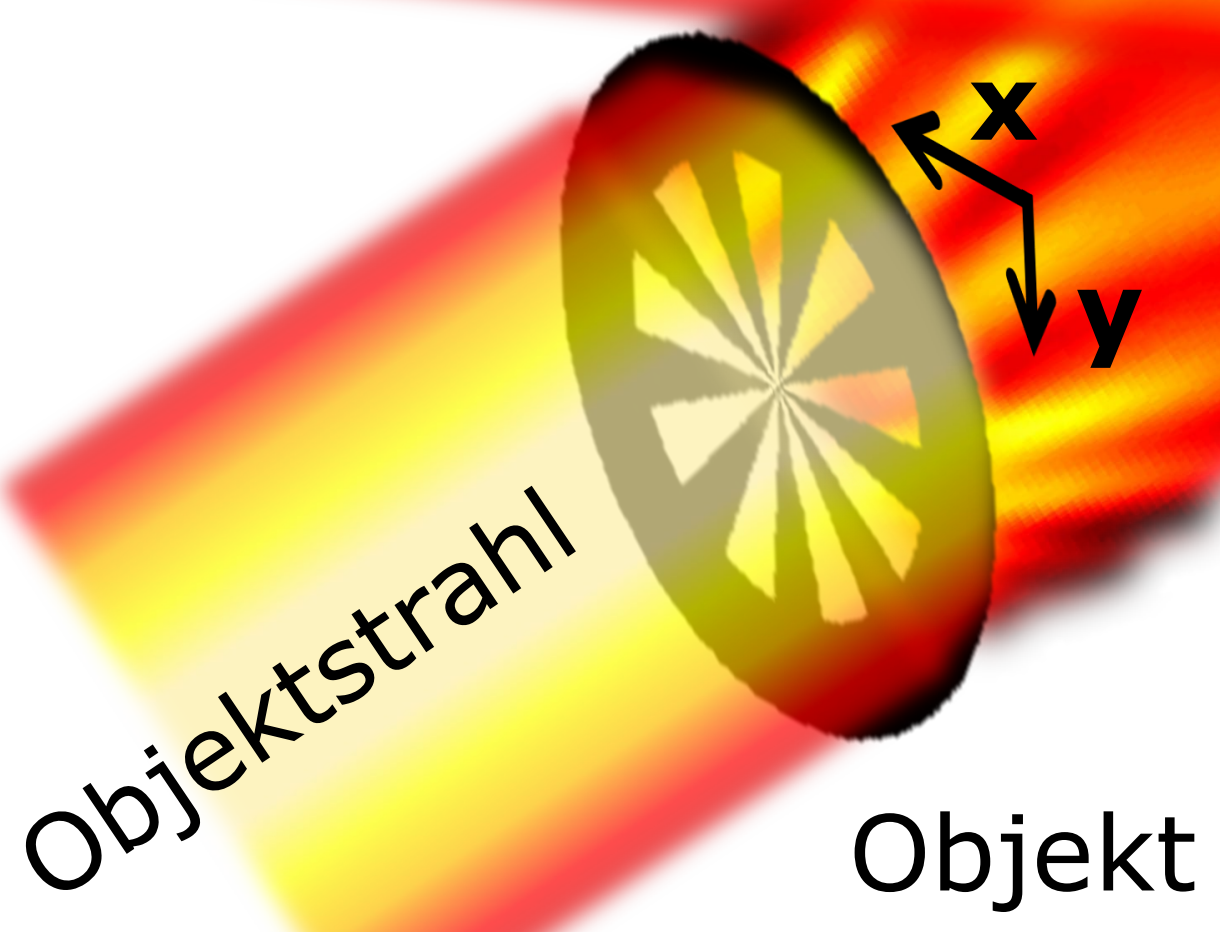

Detektorverschiebung 


\section{Detektor}

Objekt

Blende

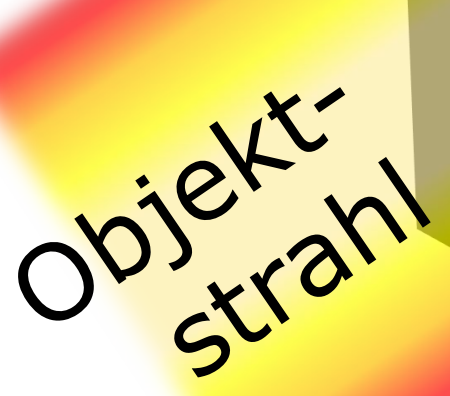

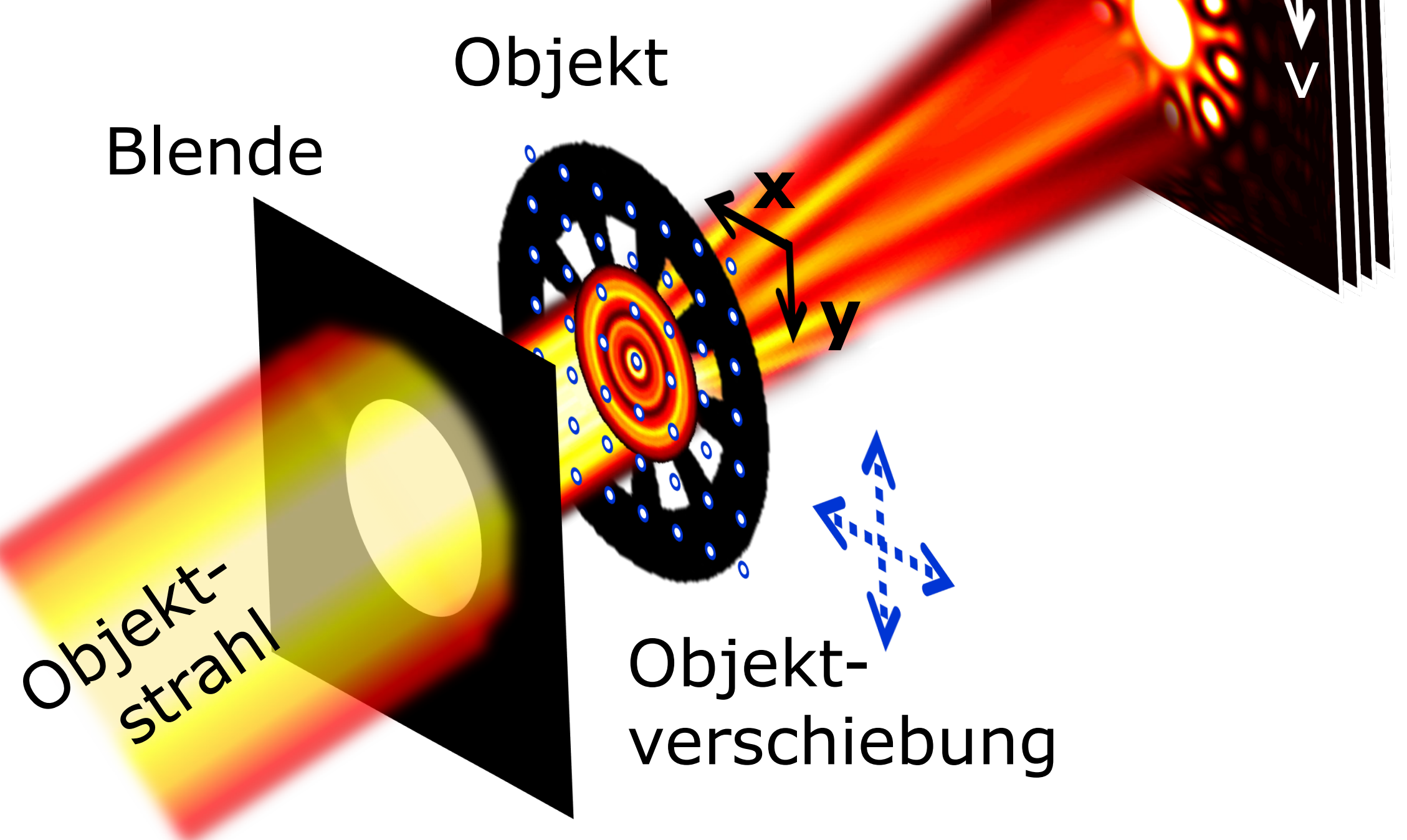


(a)

(b)

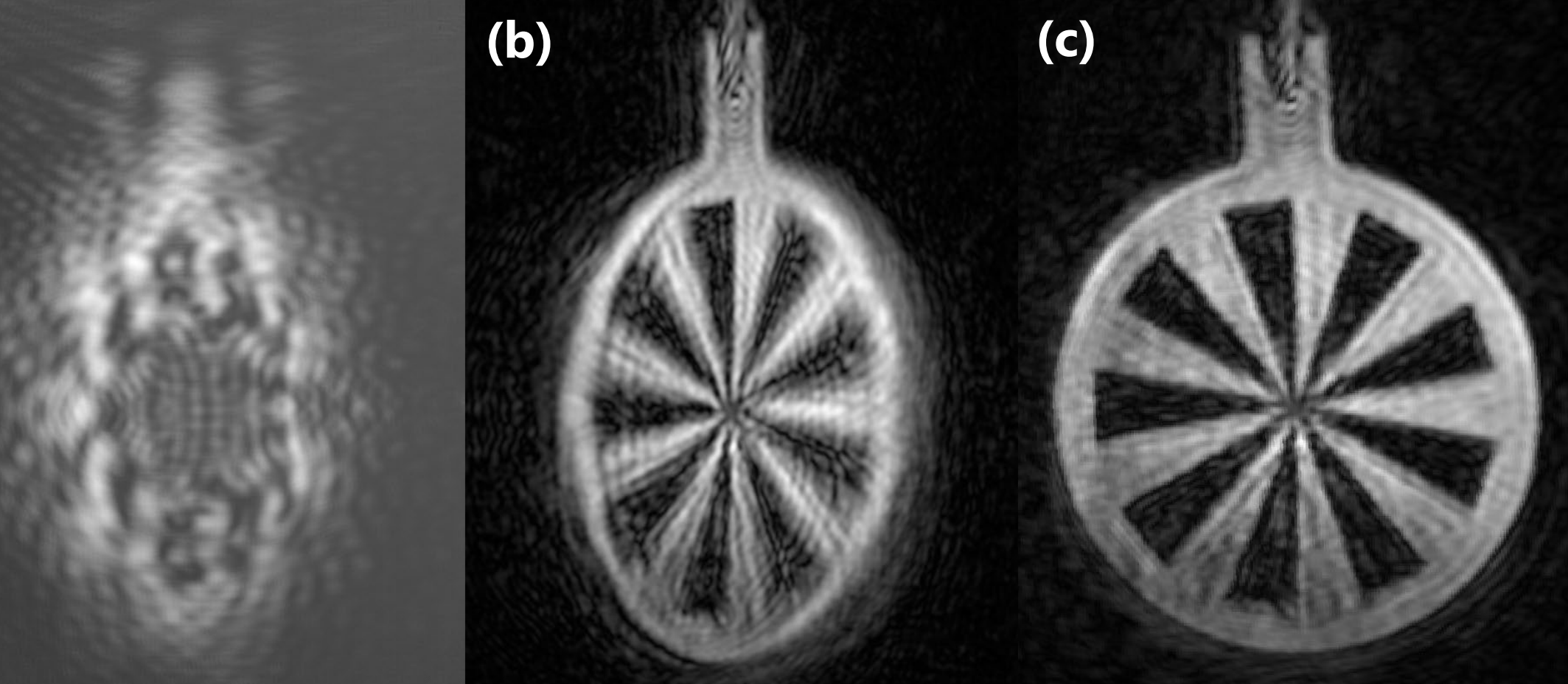

(d) 13 anयाओim

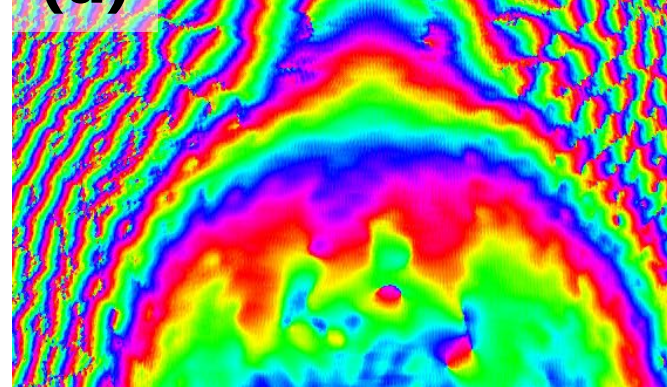

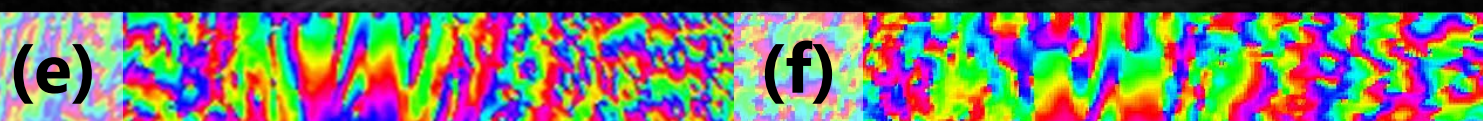
3 in

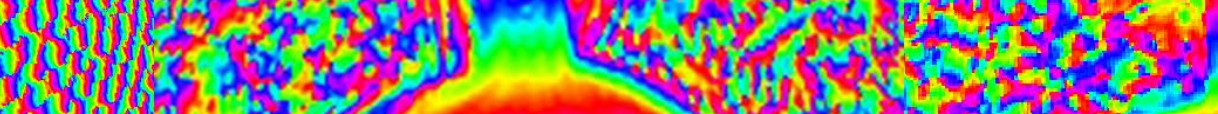

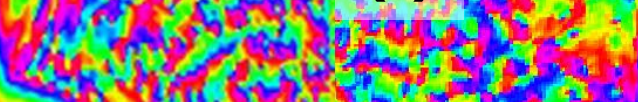

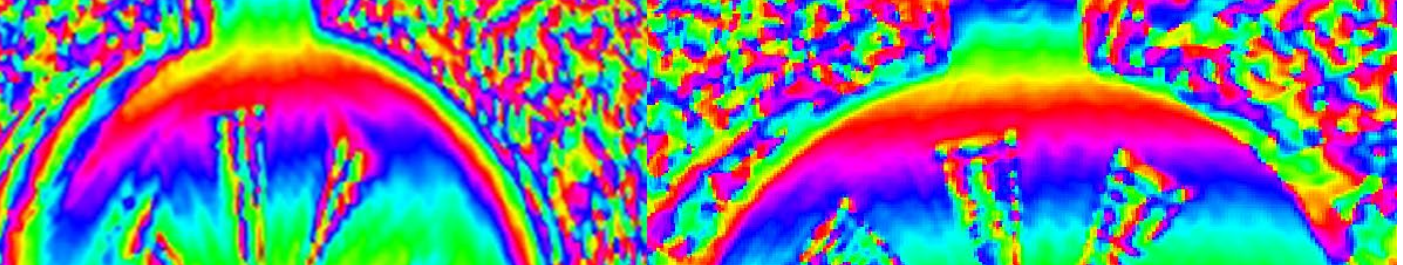
m.

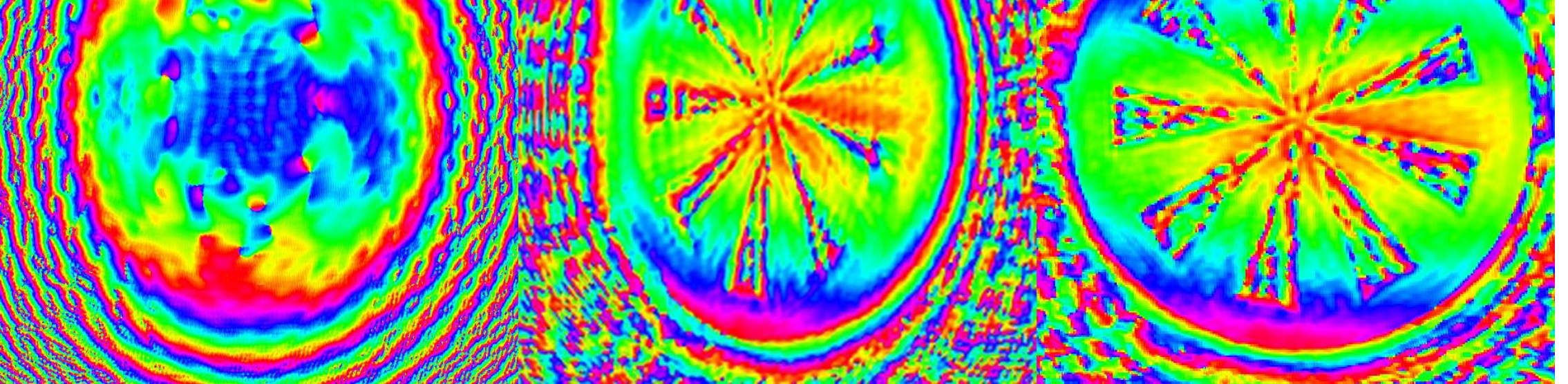



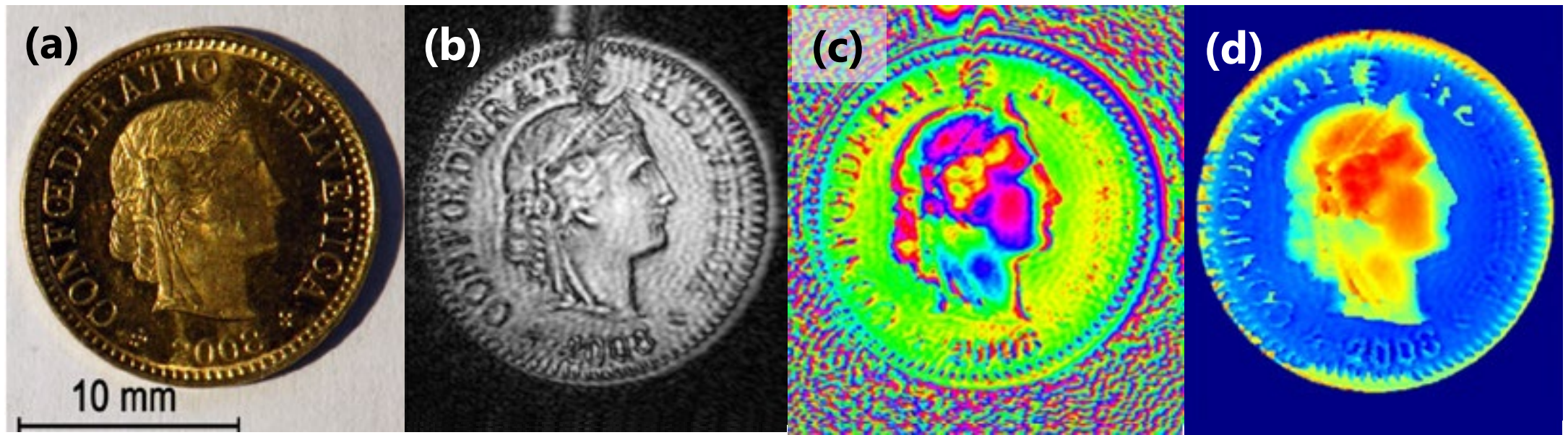

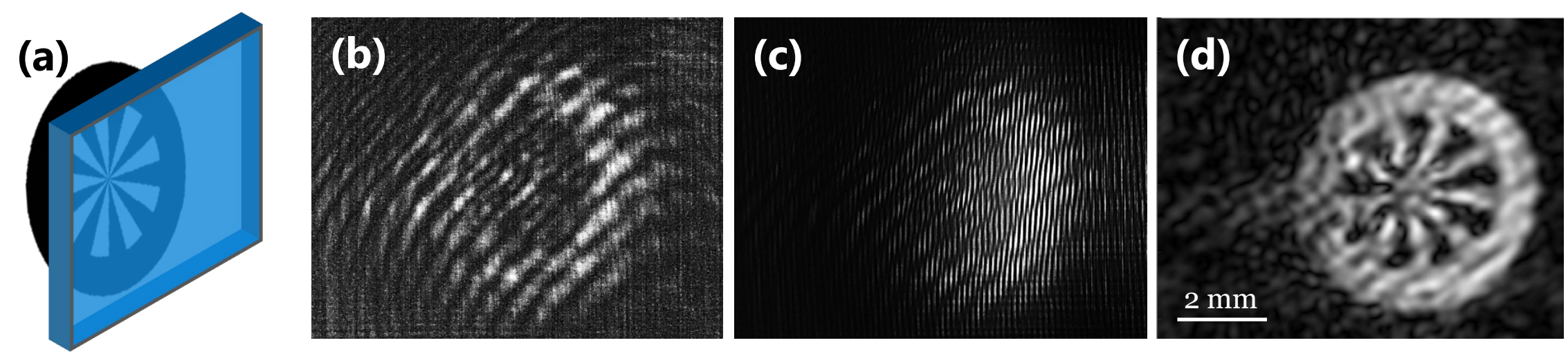
THz topography from non-metallic sample Empa
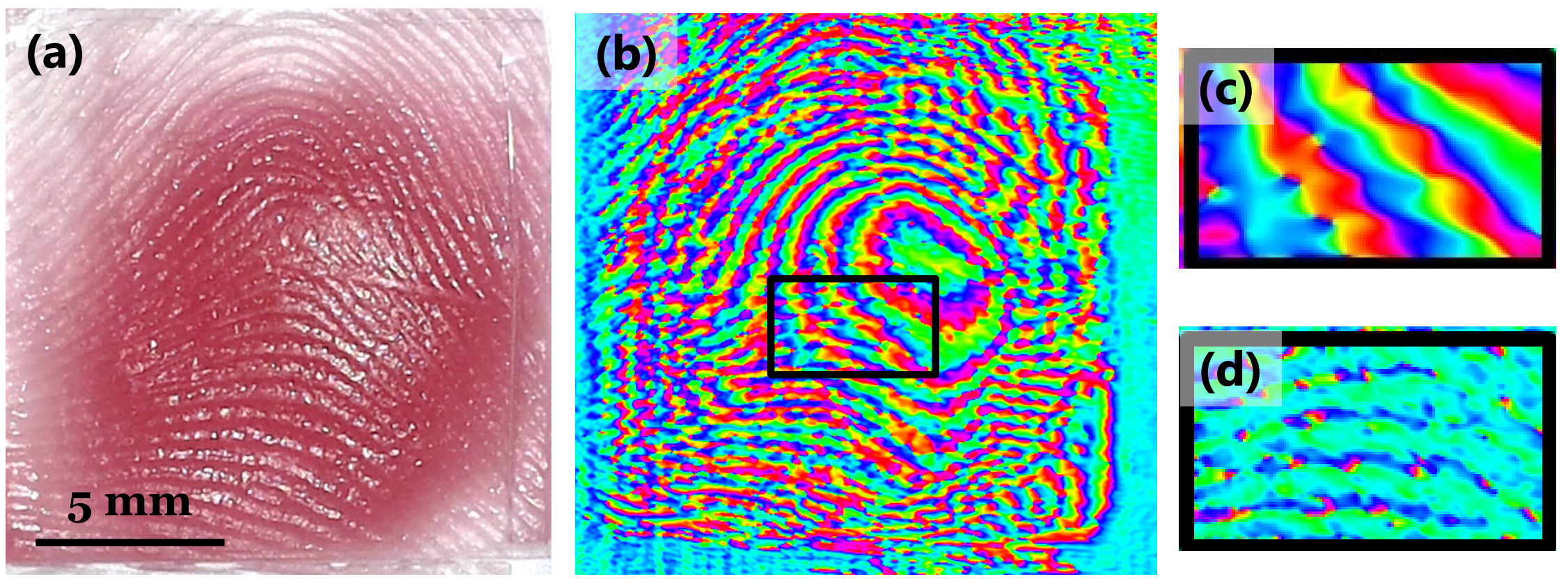